\title{
REPRESENTACIÓN MATEMÁTICA Y TÉCNICAS DE CARACTERIZACIÓN PARA CELDAS PIEZOELÉCTRICAS, UNA REVISIÓN BIBLIOGRÁFICA ${ }^{1}$
}

\author{
MATHEMATICAL REPRESENTATION AND CHARACTERIZATION TECHNIQUES FOR \\ PIEZOELECTRIC CELLS, A BIBLIOGRAPHICAL REVIEW \\ REPRESENTAÇÃO MATEMÁTICA E TÉCNICAS DE CARACTERIZAÇÃO PARA CELAS \\ PIEZOELÉCTRICAS, UMA REVISÃO BIBLIOGRÁFICA \\ Hernando Evelio Leyton Vásquez ${ }^{2}$; Néstor Raúl Roa Becerra ${ }^{3}$
}

Fecha de recibido: Febrero 05 de 2017 | Fecha de aprobado: Abril 18 de 2017

\section{Resumen}

En este artículo se expone en forma autocontenida el esquema matemático que define el modelo piezoeléctrico, el cual se emplea para describir el comportamiento físico en los materiales capaces de crear carga eléctrica bajo la influencia de fuerzas mecánicas. Adicionalmente, se presentan algunas técnicas utilizadas en la caracterización eléctrica de celdas desarrolladas en grupos de investigación en contextos nacionales e internacionales, que trabajan en el desarrollo de fuentes de energía alternativas.

Palabras clave: celdas piezoeléctricas, modelo matemático, caracterización.

1 Artículo de revisión.

2 Msc. Matemática Aplicada. Docente tiempo completo. Corporación Universitaria Minuto de Dios-UNIMINUTO. Correo electrónico: hleyton@uniminuto.edu

3 Msc. Tecnología Educativa. Docente tiempo completo. Corporación Universitaria Minuto de Dios-UNIMINUTO. Correo electrónico: nroa@uniminuto.edu 


\section{Abstract}

This article intends to describe the mathematical schema that defines the piezoelectric model in a self - contained way. This schema is used to describe the physical behavior of materials capable of creating electric charge under the influence of mechanical forces. In addition, some techniques used in the electric characterization of cells developed in research groups in national and international contexts are presented. These research groups work on the development of alternative energy sources projects.

Key words: piezoelectric Cells, Mathematical Model, Characterization.

\section{Resumo}

Neste artigo expõe-se em forma autocontenida o esquema matemático que define o modelo piezoeléctrico, o qual se emprega para descrever o comportamento físico nos materiais capazes de criar ônus elétrico baixo a influência de forças mecânicas. Adicionalmente, apresentam-se algumas técnicas utilizadas na caracterização elétrica de celas desenvolvidas em grupos de investigação em contextos nacionais e internacionais, que trabalham no desenvolvimento de fontes de energia alternativas.

Palavras-chave: celas piezoeléctricas, modelo matemático, caracterização. 


\section{INTRODUCCIÓN}

La piezoelectricidad es un fenómeno eléctrico que se presenta en algunos minerales y fue descubierto por los hermanos Curie (J. Curie, 1880; P. Curie, 1880) a finales del siglo XIX. Sin embargo, solo hasta el año de 1916 se dio a conocer su primera aplicación en el trabajo de Paul Langevin y Constantin Chilowski en la construcción de un detector ultrasónico utilizado para la medición de los fondos marinos y la detección de submarinos durante la Primera Guerra Mundial. Según la bibliografía relacionada con la generación de energía a partir de celdas piezoeléctricas, en 1944 los científicos B.M. Wool e I.P. Goldman, del Lebedev Physycal Institute, fueron los primeros en implementar un método de sinterización de cerámicas piezoeléctricas con titanato de bario $\left(\mathrm{BaTiO}_{3}\right)$ (Sharapov, 2006; Ukraine Patent No. 56930, 2011), aunque, posteriormente, se extendió al titanato zirconato de plomo (PZT), niobato de litio (LiNbO3), zirconato de plomo $\left(\mathrm{PbZrO}_{3}\right)$, titanato de plomo $\left(\mathrm{PbTiO}_{3}\right)$ y otras combinaciones (Cady, 1964). El presente artículo, de carácter introductorio a las cerámicas piezoeléctricas, surge de la revisión bibliográfica de varias fuentes documentales académicas (libros, revistas especializadas, trabajos de grado y google académico), en las que se indaga sobre cuáles son los elementos físicos y matemáticos más relevantes en el modelamiento de una celda piezoeléctrica y cómo podrían caracterizar experimentalmente después de su elaboración, con el fin de complementar la línea experimental utilizada en la elaboración de cerámicas piezoeléctricas de titanato de bario $\left(\mathrm{BaTiO}_{3}\right)$ del proyecto "Determinación de la energía producida en relación a las dimensiones de cerámicas piezoeléctricas de titanato de bario para aplicaciones en Ecosistemas Inteligentes", del grupo de investigación del Programa Tecnología en Electrónica de la Facultad de Ingeniería UNIMINUTO - Sede principal. Actualmente, de acuerdo con las actividades del proyecto antes mencionado se está realizando la evaluación del tamaño de las cerámicas piezoeléctricas que permitirá obtener una producción de energía y resistencia mecánica adecuada para aplicaciones viales.

\section{ASPECTOS PRELIMINARES DE LA PIEZOELECTRICIDAD}

La Piezoelectricidad es una propiedad física de algunos materiales no conductores cristalinos como, por ejemplo, la turmalina, el cuarzo y la sal de Rochelle (figura 1), en la que ante la variación de su estructura cristalina por una fuerza mecánica externa (tensión) genera un momento dipolar eléctrico y, con ello, un voltaje. Este proceso es llamado efecto piezoeléctrico directo (también se presenta un comportamiento inverso, es decir, que ante la aplicación de un campo eléctrico se produce una deformación), y aunque el fenómeno es perceptible de forma natural, en la industria se han creado algunas combinaciones con cristales ferroeléctricos como el titanato de bario $\left(\mathrm{BaTiO}_{3}\right)$, que pueden ser optimizados al polarizarse (Nelson, 2010; Reza, 2006).

Figura 1. Turmalina - cuarzo - sal de Rochelle

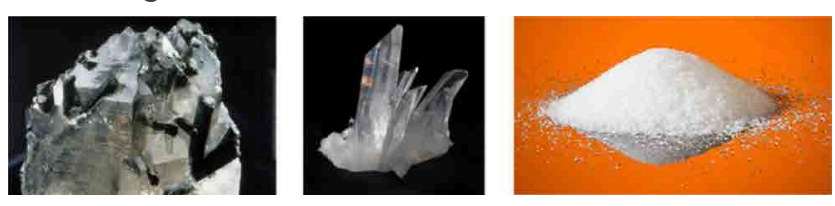

Fuente: tomado y adaptado de Nelson (2010).

En términos químicos, y como se presenta en la figura 2, las cerámicas piezoeléctricas de titanato de bario $\left(\mathrm{BaTiO}_{3}\right)$ están compuestas por cristales de perovskita, los cuales poseen una estructura cúbica con cationes grandes en sus esquinas (Bario - Ba) y en su interior, una estructura octaédrica de oxígenos con un catión más pequeño en su centro (titanio $\mathrm{Ti}$ ), que es precisamente el que permite la generación del momento magnético al ser presionado y polarizado.

Figura 2. Estructura cristalina cubica del $\mathrm{BaTiO}_{3}$

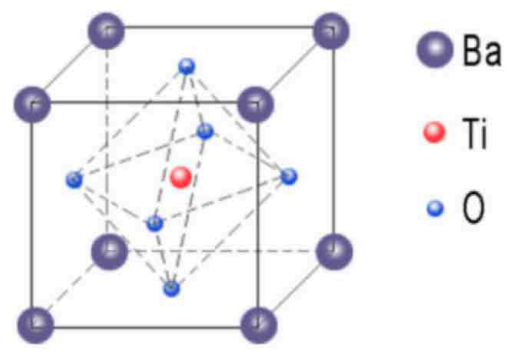

Fuente: tomado y adaptado de Wang (2010).

La cerámica se polariza sometiéndola a un campo eléctrico específico en una temperatura cercana a la temperatura de Curie (definida como la temperatura en la que los materiales ferromagnéticos cambian sus propiedades a paramagnéticos), lo cual define la dirección de la fuerza de compresión que dará la mejor respuesta en términos de voltaje (Orozco, 2015; Dineva, 2014).

El titanato de bario $\left(\mathrm{BaTiO}_{3}\right)$ y en general los cristales ferroeléctricos poseen un comportamiento 
característico en su polarización inducida cuando es aplicado sobre ellos un campo eléctrico alterno, tal fenómeno es conocido como histéresis ferroeléctrica (figura 3); de él, específicamente del área bajo la curva correspondiente a la energía disipada en forma de calor debido a las pérdidas mecánicas y dieléctricas, se puede obtener un valor que permite clasificarlos como cristales ferroeléctricos de alta potencia "hard" o de baja potencia "soft" (Celanovic, 1997).

Figura 3. Histéresis ferroeléctrica

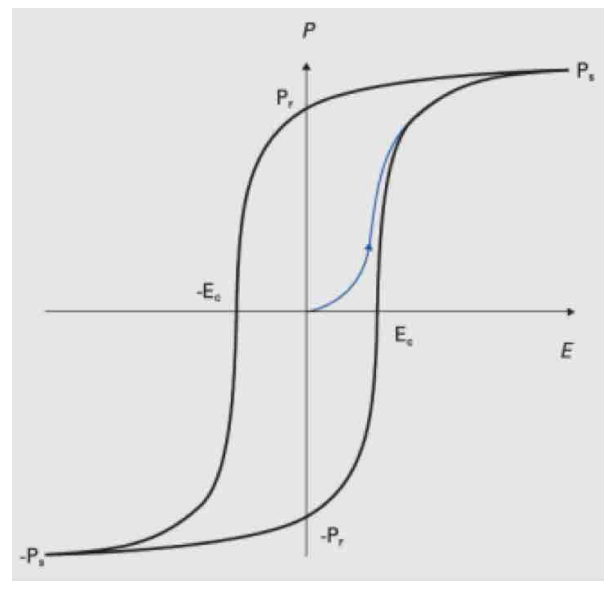

Fuente: tomado y adaptado de Celanovic (1997).

La marina de Estados Unidos (Us, 1984) ha clasificado estos cristales como Navy Type I, II, III, IV, y v. En donde el Type iv ("hard") corresponde a composiciones modificadas de titanato de bario $\left(\mathrm{BaTiO}_{3}\right)$ con menor actividad piezoeléctrica y una temperatura Curie más baja que cualquiera de las cerámicas PZT. En la siguiente tabla (tabla 1), se presenta la información ofrecida generalmente por el fabricante, en donde para este caso específico, los valores se encuentran diferenciados por el tamaño de sus granos $(300,600$ y 1300).

Tabla 1. Propiedades de los materiales piezoeléctricos Channel Industries, inc.

\begin{tabular}{|c|c|c|c|c|}
\hline \multirow{2}{*}{\multicolumn{2}{|c|}{\begin{tabular}{|l} 
\\
Material Reference \\
Numbers Channel \\
Industries, Inc. \\
U.S. Navy
\end{tabular}}} & \multicolumn{3}{|c|}{ BARIUM TITANATE } \\
\hline & & \multirow{2}{*}{$\begin{array}{r}300 \\
.46\end{array}$} & \multirow{2}{*}{$\begin{array}{r}600 \\
.39\end{array}$} & \multirow{2}{*}{$\begin{array}{c}1300 \\
\text { Navy IV } \\
.45\end{array}$} \\
\hline \multirow{4}{*}{$\begin{array}{l}\text { Coupling } \\
\text { Coefficients }\end{array}$} & $\mathrm{k}_{33}$ & & & \\
\hline & $k_{31}$ & -.19 & -.16 & -.18 \\
\hline & $\mathrm{k}_{15}$ & .46 & .39 & .45 \\
\hline & $k_{p}$ & -.32 & -.27 & -.30 \\
\hline \multicolumn{5}{|c|}{ Piezoelectric Constants } \\
\hline $\mathrm{d}_{33}$ & $10^{-12} \mathrm{~m} / \mathrm{V}$ & 145 & 82 & 145 \\
\hline
\end{tabular}

\begin{tabular}{|c|c|c|c|c|}
\hline \multirow{2}{*}{\multicolumn{2}{|c|}{$\begin{array}{l}\text { Material Reference } \\
\text { Numbers Channel } \\
\text { Industries, Inc. } \\
\text { U.S. Navy }\end{array}$}} & \multicolumn{3}{|c|}{ BARIUM TITANATE } \\
\hline & & \multirow{2}{*}{$\begin{array}{r}300 \\
-58\end{array}$} & \multirow{2}{*}{$\begin{array}{l}600 \\
-33\end{array}$} & \multirow{2}{*}{$\begin{array}{c}1300 \\
\text { Navy IV } \\
-56 \\
\end{array}$} \\
\hline$d_{31}$ & “ & & & \\
\hline$d_{15}$ & “ & 245 & 150 & 245 \\
\hline $\mathrm{g}_{33}$ & $10^{-12} \mathrm{Vm} / \mathrm{N}$ & 13.1 & 16.8 & 12.2 \\
\hline $\mathrm{g}_{31}$ & “ & -5.2 & -6.8 & -4.7 \\
\hline $\mathrm{g}_{15}$ & “ & 20.5 & 29.8 & 19.1 \\
\hline \multicolumn{5}{|c|}{ Free Dielectric Constants } \\
\hline \multicolumn{2}{|c|}{$\mathrm{K}_{3}^{\top}$} & 1250 & 625 & 1350 \\
\hline \multicolumn{2}{|c|}{$\mathrm{K}_{1}^{\top}$} & 1350 & 570 & 1450 \\
\hline
\end{tabular}

Fuente: adaptación propia.

Los datos presentados en la tabla, aunque son solo un ejemplo, nos sirve para establecer un estimado de los parámetros que se utilizarán más adelante cuando se defina un modelo matemático para el análisis de los fenómenos piezoeléctricos y la determinación de las características eléctricas y mecánicas de las celdas piezoeléctricas, aunque en ese punto también dependerá de los protocolos y técnicas implementados en su elaboración.

\section{MODELO MATEMÁTICO}

Desde 1987 las relaciones constitutivas de un material piezoeléctrico (para pequeñas condiciones de campo), fueron establecidas por el Instituto de Electricidad y Electrónica (Institute of Electrical and Electronic, IEEE) en términos de constantes piezoeléctricas, de permitividad y de deformación elástica como:

$$
\left(\begin{array}{l}
D_{1} \\
D_{2} \\
D_{3} \\
\varepsilon_{1} \\
\varepsilon_{2} \\
\varepsilon_{3} \\
\varepsilon_{4} \\
\varepsilon_{5} \\
\varepsilon_{6}
\end{array}\right)=\left(\begin{array}{ccccccccc}
e_{11}^{\sigma} & 0 & 0 & 0 & 0 & 0 & 0 & d_{15}^{d} & 0 \\
0 & e_{22}^{\sigma} & 0 & 0 & 0 & 0 & d_{24}^{d} & 0 & 0 \\
0 & 0 & e_{33}^{\sigma} & d_{31}^{d} & d_{32}^{d} & d_{33}^{d} & 0 & 0 & 0 \\
0 & 0 & d_{31}^{c} & s_{11} & s_{12} & s_{13} & 0 & 0 & 0 \\
0 & 0 & d_{32}^{c} & s_{12} & s_{22} & s_{23} & 0 & 0 & 0 \\
0 & 0 & d_{33}^{c} & s_{13} & s_{23} & s_{33} & 0 & 0 & 0 \\
0 & d_{24}^{c} & 0 & 0 & 0 & 0 & s_{44} & 0 & 0 \\
d_{15}^{c} & 0 & 0 & 0 & 0 & 0 & 0 & s_{55} & 0 \\
0 & 0 & 0 & 0 & 0 & 0 & 0 & 0 & s_{66}
\end{array}\right)\left(\begin{array}{c}
E_{1} \\
E_{2} \\
E_{3} \\
\sigma_{11} \\
\sigma_{22} \\
\sigma_{33} \\
\sigma_{23} \\
\sigma_{31} \\
\sigma_{12}
\end{array}\right)
$$

Escrita en forma resumida como:

$$
\begin{gathered}
\varepsilon_{k}=d_{j k}^{c} E_{j}+s_{k m}^{E} \sigma_{i} \\
D_{i}=e_{i j}^{\sigma} \cdot E_{j}+d_{i m}^{d} \sigma_{i}
\end{gathered}
$$

Donde el vector de desplazamiento eléctrico $D$ $\left(\frac{\text { Coulomb }}{m^{2}}\right)$ es de tamaño $(3 \times 1)$, es el vector deformación (adimensional) de tamaño $(6 \times 1), E$ es el vector de campo eléctrico aplicado $\left(\frac{\text { voltios }}{m}\right)$ de tamaño $(3 \times 1)$ y $\sigma_{m}$ 
es el vector tensión $\left(\frac{N}{m^{2}}\right)$ de tamaño $(6 \times 1)$. Las constantes piezoeléctricas son la constante dieléctrica $e_{i j}^{\sigma}$ $\left(\frac{\text { Farad }}{m}\right)$ de tamaño $(3 \times 3)$, la deformación elástica $s_{k m}^{E}$ $\left(\frac{m^{2}}{N}\right)$ de tamaño $(6 \times 6)$ y los coeficientes piezoeléctricos o también llamadas constantes piezoeléctricas $d_{i m}^{d}$ y $d_{j k}^{c}\left(\frac{\text { Coulomb }}{N} o \frac{m}{\text { voltios }}\right)$ de tamaño $(3 \times 6)$ y $(6 \times 3)$ respectivamente (los superíndices y se han añadido para diferenciar entre el efecto piezoeléctrico inverso "converse" y el directo "direct", aunque en la práctica estos coeficientes sean numéricamente iguales) (IEEE,1987). Asumiendo la nomenclatura utilizada en Moheimani (2006) (figura 4):

Figura 4. Nomenclatura de los ejes

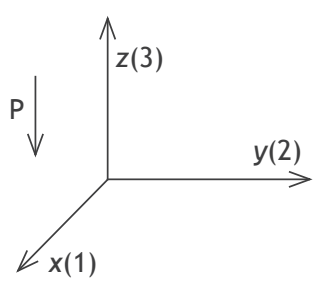

\begin{tabular}{|c|c|}
\hline No & Eje \\
\hline 1 & $x$ \\
\hline 2 & $y$ \\
\hline 3 & $z$ \\
\hline 4 & Corte alrededor de $x$ \\
\hline 5 & Corte alrededor de $y$ \\
\hline 6 & Corte alrededor de $z$ \\
\hline
\end{tabular}

Fuente: tomado y adaptado de Moheimani (2006).

Y tomando el modelo específicamente para el caso de una celda piezoeléctrica utilizada para la generación de carga, donde el campo eléctrico externo aplicado es cero, las ecuaciones en términos de constantes piezoeléctricas $d_{i j}$ que son las que establecen una proporcionalidad entre la generación de cargas y la tensión mecánica aplicada medidas a temperatura y campo eléctrico constante, se convierten, según Sirohi (2000) y Moreno (2016) en:

$$
\left[\begin{array}{l}
D_{1} \\
D_{2} \\
D_{3}
\end{array}\right]=\left[\begin{array}{cccccc}
0 & 0 & 0 & 0 & d_{15} & 0 \\
0 & 0 & 0 & d_{15} & 0 & 0 \\
d_{31} & d_{31} & d_{33} & 0 & 0 & 0
\end{array}\right]\left[\begin{array}{c}
\sigma_{1} \\
\sigma_{2} \\
\sigma_{3} \\
\sigma_{4} \\
\sigma_{5} \\
\sigma_{6}
\end{array}\right]
$$

De lo anterior, y del hecho de que casi todas las celdas piezoeléctricas se diseñan de forma tal que su máximo desempeño se dé cuando sobre ellas se aplica una tensión de manera perpendicular (eje $z(3)$ ), podemos concluir que el coeficiente más representativo en este modelo es el $d_{33}$ que representa el coeficiente de carga piezoeléctrica y establece la relación entre la carga que fluye en la dirección cuando se aplica sobre ella una tensión también en esta dirección, siempre que el material está libre de cualquier otra tensión.

Así pues, es posible estimar la cantidad de carga eléctrica generada en el material en términos del desplazamiento eléctrico $D$ como Moheimani (2006) y Montero (1991):

$$
q=\iint\left[\begin{array}{lll}
D_{1} & D_{2} & D_{3}
\end{array}\right]\left[\begin{array}{l}
d A_{1} \\
d A_{2} \\
d A_{3}
\end{array}\right]
$$

Donde $d A_{1}, d A_{2}$ y $d A_{3}$ son las componentes del área del electrodo en los planos 1-2, 1-3 y 2-3 respectivamente y con la idea de aplicar tensión solo en forma perpendicular. En conclusión, la carga recogida depende solo del área del electrodo perpendicular al desplazamiento $D$.

\section{CARACTERIZACIÓN DE UNA CELDA PIEZOELÉCTRICA}

Otra manera de abordar el estudio de las celdas piezoeléctricas, es determinando un modelo eléctrico equivalente que permita obtener algunas de sus propiedades piezoeléctricas y elásticas cuando ya están elaboradas. Uno de los parámetros más útiles para lograr esto es el valor de la frecuencia de resonancia $f_{r}$, valor que se obtiene experimentalmente utilizando el hecho de que las cerámicas poseen una frecuencia de resonancia en la que su impedancia es mínima y su resonancia es mayor a la medida en cualquier otra frecuencia; el fenómeno contrario se presenta a una frecuencia que es llamada anti resonante $f_{a}$. La idea se presenta en la figura 5 (Jordan, 2001).

Figura 5. Variación de la impedancia en función de la frecuencia

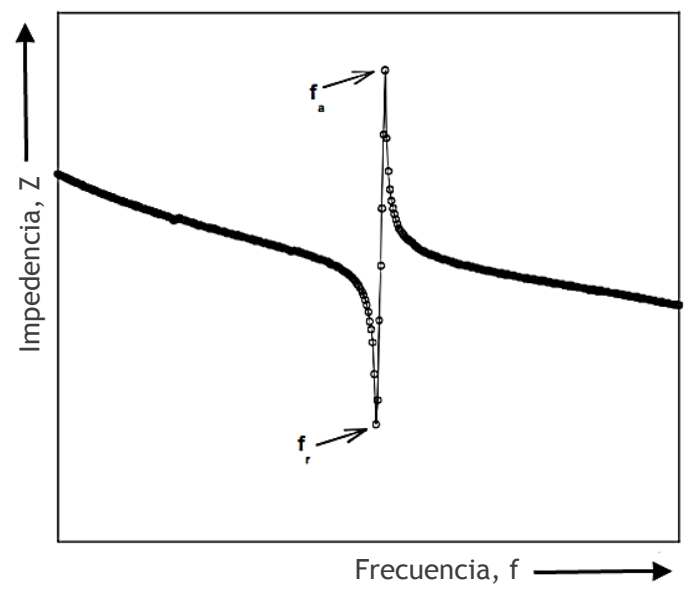

Fuente: tomado y adaptado de Jordan (2001).

La frecuencia de resonancia $f_{r}$, por ejemplo, para una celda circular plana utilizada como resonador piezoeléctrico, se obtiene de la división del valor de la constante de frecuencia para vibraciones planas $N_{P}^{E}$ y el diámetro del disco, gracias a que el módulo 
de la impedancia y de la admitancia para valores cercanos a la frecuencia de resonancia y antiresonancia es puramente óhmica como se muestra en la figura 6 (González, 2013); figura en la que también es posible determinar el factor de calidad $Q$ (Arnau, 2004).

Figura 6. Curva de resonancia de un transductor piezoeléctrico

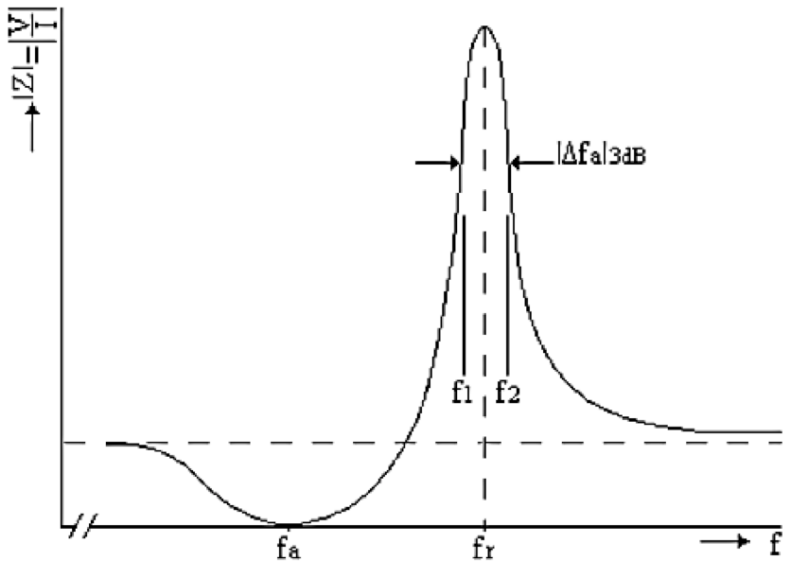

Fuente: tomado y adaptado de Arnau (2004).

$$
\begin{gathered}
f_{r}=\frac{N_{P}^{E}}{D} \\
Q=\frac{f_{r}}{f_{2}-f_{1}}
\end{gathered}
$$

En IEEE (1987) y Jordan (2001) se propone que en resonancia, la cerámica puede ser modelada como un circuito RLC serie (resistencia, bobina, condensador) en paralelo con un condensador. El modelo es denominado modelo Van Dyke (figura 7), aunque en otras referencias (Jin, 2011; Choi, 2013; Wu, 2003, entre otros), es llamado Butterworth-Van Dike (BVD) y será el utilizado en el proyecto de investigación antes mencionado.

Figura 7. Modelo Van Dyke

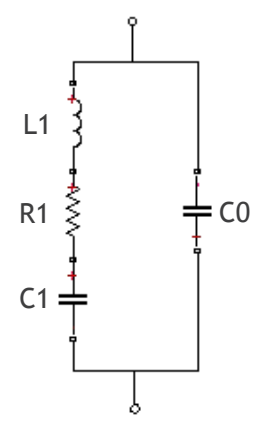

Fuente: elaboración propia.

Existen algunas pequeñas variaciones al modelo, como, por ejemplo, la trabajada por Sherrit (1997) en donde se elimina la resistencia $R_{1}$, o los modelos Butterworth-Van Dike Modificado (MBVB), trabajado por Cernosek (1998) y Zalazara (2010), y el Butterworth-Van Dike extendido (EBVD), de Torres, en donde básicamente se adicionan más condensadores en paralelo.

\section{Primera técnica experimental (Zalazara, 2010)}

Para el modelo más simple, los valores de las componentes pueden ser calculadas con el dato de los parámetros de los materiales y sus características geométricas como:

$$
\begin{gathered}
C_{0}=\frac{p_{p} A_{e}}{h_{p}} \\
C_{1}=\frac{8 C_{0} K_{e}^{2}}{\pi^{2}} \\
L_{1}=\frac{1}{w_{0}^{2} C_{1}} \\
R_{1}=\frac{\eta_{p}}{c_{p} C_{1}}
\end{gathered}
$$

Donde $p_{p}$ es la permitividad del piezoeléctrico, $A_{e}$ el área del electrodo, el espesor del piezoeléctrico, $\omega_{0}$ la frecuencia angular característica, $\eta_{p}$ la viscosidad del piezoeléctrico y $K_{e}$ la constante de acoplamiento 
electromecánico, que es un valor muy importante ya que se encarga de establecer la efectividad con la que se convierte la acción mecánica en energía eléctrica (Gutiérrez, 2010).

Otros valores significativos que se deben tener en cuenta al momento de elaborar las celdas piezoeléctricas son: el factor de calidad mecánico $Q_{m}$ y el factor de disipación dieléctrico . Estos valores son los más importantes en la definición de las posibles aplicaciones dinámicas del material, ya que a través de ellos es posible determinar las pérdidas de energía en el proceso de transducción (Pereira, 2009; Sokhanvar, 2007; Dubois, 1999; Wingqvist, 2000). En Measurement Specialties (1999) aparece otro ejemplo de valores tenidos en cuenta en la elaboración de celdas piezoeléctricas con Titanato de Bario $\mathrm{BaTiO}_{3}$, como la densidad $5.7 \times 10^{3}\left[\frac{\mathrm{Kg}}{\mathrm{m}^{3}}\right]$, permitividad relativa 1700 y las constantes $d_{31}=78 \times 10^{-12}\left[\frac{\mathrm{C}}{\mathrm{N}}\right]$, $g_{31}=5 \times 10^{-3}\left[\frac{V \cdot m}{N}\right]$ y $k_{31}=21 \%$ a $1 \mathrm{KHz}$.

\section{Segunda técnica experimental} (González, 2009)

Otra manera de obtener el valor de los elementos que se utilizan en el modelo Van Dyke es utilizando la celda piezoeléctrica como resonador, midiendo de ella para unos montajes específicos los valores de la frecuencia de resonancia $f_{r}$, antiresonancia $f_{a}$, resistencia de la celda a la frecuencia $R_{1}$ de resonancia y la capacitancia a frecuencias muy por debajo de la $f_{r}$.

En la figura 8 se presenta el montaje que permite medir los parámetros antes mencionados, donde el valor de $C_{0}$ por poseer una impedancia muy alta tiene un efecto despreciable sobre el modelo. Es necesario tener en cuenta que la resistencia en serie del circuito debe ser mucho mayor que la impedancia del resonador a la $f_{r}$, que es donde el voltímetro da una lectura mínima $V_{2}$, de modo tal que el valor de $R_{1}$ puede ser obtenido de:

$$
V_{2}=\frac{R_{1}}{R+R_{1}} V_{1}
$$

Figura 8. Circuito para la medición de $f_{\mathrm{r}}$

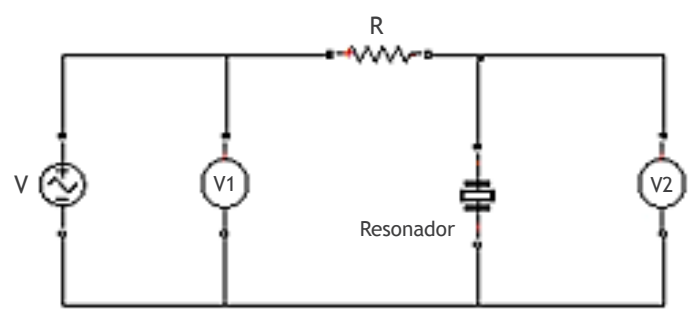

Fuente: elaboración propia.

De manera similar y con la utilización del montaje presentado en la figura 9, la frecuencia de antiresonancia $f_{a}$ puede ser medida bajo la condición que $R_{2}$ sea mucho menor que la impedancia del resonador a $f_{a}$ (González, 2009). Cuando se mide la capacidad del resonador a una frecuencia muy por debajo de la frecuencia de resonancia $(1 \mathrm{kHz})$, se encuentra un valor $C_{l f}$ que es la suma de $C_{0}$ y $C_{1}$ :

$$
C_{l f}=C_{0}+C_{1}
$$

Figura 9. Circuito para la medición de $f_{\mathrm{a}}$

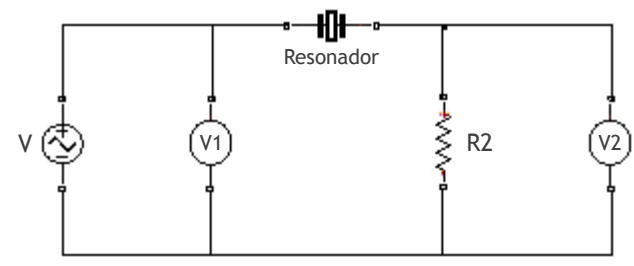

Fuente: elaboración propia.

Con los resultados medios de los montajes anteriores, los valores del circuito equivalente se hallan al desarrollar:

$$
\begin{gathered}
\frac{C_{0}}{C_{1}}=\frac{f_{r}^{2}}{f_{a}^{2}-f_{r}^{2}} \\
L_{1}=\frac{1}{w_{r}^{2} C_{1}} \\
C_{1}=\frac{C_{l f}}{1+\frac{C_{0}}{C_{1}}} \\
Q_{m}=\frac{1}{w_{r} \cdot C_{m} \cdot R_{m}}
\end{gathered}
$$

Con factor de mérito mecánico o factor de calidad. 
Tercera técnica experimental (Cifuentes, 2013)

Cuando la celda se utiliza como generador de energía eléctrica, se puede presentar un modelo alternativo representado por un circuito $\mathrm{RC}$ como se muestra en la figura 10.

Figura 10. Circuito equivalente para un generador piezoeléctrico

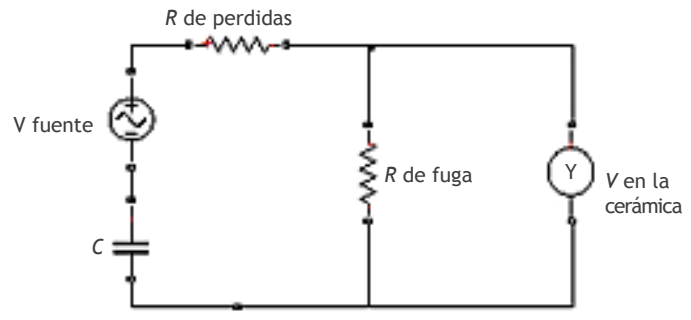

Fuente: tomado y adaptado de Cifuentes (2013).

Dónde $C_{\text {stack }}$ se define como la capacitancia de apilamiento, $R_{\text {loss }}$ la resistencia por pérdidas, $R_{\text {leakage }}$ resistencia por fuga (generalmente $10^{6} \Omega$ ) $V_{a}$ y el voltaje en circuito abierto producido en el piezoeléctrico como resultado de una fuerza aplicada. En este caso los valores del circuito equivalente se obtienen al desarrollar (Keawboonclinay, 2003):

$$
C_{\text {stack }}=\frac{\varepsilon_{0} \varepsilon_{r} A}{h_{\text {piezo }}}
$$

Con $\varepsilon_{0}$ la permisividad libre del espacio $8.85 \times 10^{-12}$ $\left[\frac{F}{m}\right]$, permisividad relativa del material piezoeléctrico, $A$ el área transversal del piezoeléctrico y $h_{\text {piezo }}$ su espesor.

La resistencia en serie con la fuente $R_{\text {loss }}$, representa la pérdida dieléctrica interna del material y se calcula como:

$$
R_{\text {loss }}=\frac{\tan \delta}{\omega \cdot C_{\text {stack }}}
$$

Con $\omega$ la frecuencia angular de operación del generador y $\tan \delta$ el factor de disipación dieléctrico. La energía eléctrica almacenada en el piezoeléctrico como resultado de la compresión mecánica se obtiene con:

$$
W_{\text {eléctrica }}=\frac{q^{2}}{2 C_{\text {stack }}}
$$

$$
q=C_{\text {stack }} V_{a}
$$

que corresponde a la carga eléctrica almacenada en el material. Finalmente, el voltaje generado $V_{a}$ se calcula como:

$$
V_{a}=\frac{k_{33} \cdot F \cdot h_{\text {piezo }}}{A}\left(0.5 Y \varepsilon_{0} \varepsilon_{r}\right)^{-\frac{1}{2}}
$$

Donde es la fuerza aplicada y el módulo de Young del piezoeléctrico que oscila entre los 108 - $120 \mathrm{GPa}$ (Dent, 2007), generalmente se desea que sea lo más bajo posible para que el voltaje generado sea mayor.

\section{CONCLUSIONES}

A partir de la revisión de referencias, en todas ellas se establece como punto básico de inicio para el estudio de la piezoelectricidad el modelo establecido por la IEEE, y, aunque no siempre se desarrollan exhaustivamente, reconocen su importancia en el proceso de identificar y establecer la influencia de cada uno de sus elementos constitutivos en la obtención del mayor rendimiento en la elaboración y puesta a prueba mediante la experimentación en los laboratorios. La revisión bibliográfica permitió destacar las tres técnicas de caracterización eléctrica más utilizadas en grupos de investigación en proyectos de diferentes universidades a nivel local e internacional antes de realizar el trabajo experimental (Maryland, Politécnica de Valencia, Carlos III de Madrid, Nacional de Entre Ríos (Argentina), Del Cauca, Escuela de Ingeniería de Antioquia, entre otras). En todas se logra reducir el comportamiento de la celda a un circuito eléctrico en donde cada uno de los valores de los elementos es obtenido de parámetros específicos de los materiales (valores proporcionados por el vendedor), y de características geométricas propias de cada celda, las cuales se encuentran relacionadas directamente con las posibles aplicaciones dinámicas del material para los procesos de transducción. Su principal diferencia radica en la disposición que tienen los elementos dentro del circuito, ya que de ella se establecen características medibles que definen su utilización para generación eléctrica, como sensor o como resonador. Por último, es importante mencionar que dentro del proyecto de investigación del Programa Tecnología en Electrónica de la Facultad de Ingeniería UNIMINUTO, la técnica para caracterizar las cerámicas piezoeléctricas es la que utiliza el modelo Van Dyke y funciona de manera óptima, debido a su 
facilidad de implementación en el laboratorio y la medición de las frecuencias de resonancia, antiresonancia y las características geométricas de la celda.

\section{REFERENCIAS}

Arnau, A. (2004). Piezoelectric Transducers and Applications. Springer.

Cady, W. G. (1964). Piezoelectricity: An Introduction to the Theory and Applications of Electromechanical Phenomena in Crystals. E.U.: Dover Press.

Celanovic, N., \& Goldfarb, M. (1997). A lumped parameter electromechanical model for describing the nonlinear behaviour of piezoelectric actuators. Journal of Dynamic Systems, Measurement, and Control.

Cernosek, R. (1998). Comparison of Lumped Element and Transmission Line Models for Thickness Shear Mode Quartz Resonator Sensors. IEEE transactions on ultrasonics, ferroelectrics, and frequency control, 45(5).

Channel Industries, I. (2014). Properties of Piezoelectric Ceramics. Catalogo. Santa Barbara, CA 93111. USA.

Choi, H., Jung, H., Chen, R., \& Shung, K. (2013). Harmonic distortion reduction technique of the power amplifier for very high frequency ultrasonic transducer applications. Ultrasonics Symposium (IUS), IEEE International.

Cifuentes, J. (2013). Baldosa Piezoeléctrica Para Alimentar Sistemas De Iluminación De Bajo Consumo. Envigado - Colombia: Escuela De Ingeniería De Antioquia .

Córdoba, M. (2013). Estudio de la respuesta piezoeléctrica en PVDF. Recuperado de https://e-archivo.uc3m.es/PFC_Luis_Miguel_ Cordoba_Casado.pdf

Curie, J., \& Curie, P. (1880). l'électricité polaire dans les cristaux hemiedres a faces inclinees. France: Comptes Rendus de l'Académie des Sciences.

Curie, P., \& Curie, J. (1880). Development by pressure of polar electricity in hemihedral crystals with inclined faces. Bull. Soc. Min. de France.
Dent, A. C., Bowen, C. R., Stevens, R., Cain, M., \& Stewart, M. (2007). Effective elastic properties for unpoled barium titanate. Journal of the European Ceramic Society, 3739-3743.

Dineva, P., Gross, D., Müller, R., \& Rangelov, T. (2014). Dynamic Fracture of Piezoelectric Materials. Springer.

Dubois, M. P. (1999). Properties Of Aluminum Nitride Thin Films For Piezoelectric Transducers And Microwave Filter Applications. Applied Physics Letters, 74(20).

González, A., \& Cesari, R. (2009). Materiales Piezoeléctricos y Efecto Piezoeléctrico. Cátedra De Tecnología Electrónica. Mendoza, Argentina: Universidad Tecnológica Nacional.

González, N., Ferri, M., Jiménez, N., Camarena, F., Picó, R., Redondo, J., \& Roig, B. (2013). Simulación numérica de una cerámica piezoeléctrica. Modelling in Science Education and Learning - Universitat Politècnica de València, 6(12).

Gutiérrez, F. (2010). Principios de Piezoelectricidad. Innovaciòn y Experiencias Educativas.

IEEE, I. O. (1987). Standard on Piezoelectricity. Norma. New York.

Jin, H., Dong, S., Luo, J., \& Milne, W. I. (2011). Generalised Butterworth-Van Dyke equivalent circuit for thin-film bulk acoustic resonator. Electronics Letters, 47(7), 424-426.

Jordan, T., \& Ounaies, Z. (2001). Piezoelectric Ceramics Characterization. Hampton, Virginia: National Aeronautics and Space Administration NASA.

Keawboonclinay, C., \& Engel, T. G. (2003). Electrical Power Generation Characteristics of Piezoelectric Generator Under Quasi-Static and Dynamic Stress Conditions. IEEE Transactions On Ultrasonics, Ferroelectrics, And Frequency Control, 1377-1382.

Measurement Specialties, I. (1999). Piezo Film Sensors-Technical Manual.

Moheimani, S., \& Fleming, A. J. (2006). Piezoelectric Transducers for Vibration Control and Damping. London: Springer. 
Montero, F. R. (1991). Normativas para la caracterización de cerámicas piezoeléctricas. Boletín Sociedad Española De Cerámica Y Vidrio, 338 - 341.

Moreno, J., Fernández, J., Ochoa, P., Ceres, R., Calderón, L., Rocon, E., \& Pons, J. L. (2016). Aplicación de sensores piezoeléctricos cerámicos a la caracterización biomecánica. Boletín de la sociedad española de Cerámica y Vidrio.

Nelson, W. G. (2010). Structure, Properties an Aplications. New York: Nova Science Publishers.

Orozco, E. (2015). Diseño de un Colector de Energía Piezoeléctrico (Energy Harvesting) Mediante Optimización Topológica que Maximice la Transformación de Energía Mecánica en Eléctrica Generada por un Ser Humano al Caminar. Medellín: Universidad Nacional de Colombia.

Pereira, A. (2009). Cerámicas piezoeléctricas: funcionamiento y propiedades. Brasil: ATCP, Engenharia Física.

Reza, S. O., Andrew, M., \& Fleming, J. (2006). Piezoelectric Transducters for Vibration Control. London: Springer.

Sharapov, V. M. (2011). Ukraine Patent, (56930).

Sharapov, V. M., \& Musienko, M. P. (2006). Piezoelectric Sensors. Moscow: Technosphera.

Sherrit, S., Wiederick, H., \& Mukherjee, B. K. (1997). Accurate Equivalent Circuits for Unloaded Piezoelectric Resonators. IEEE Ultrasonics Symposium Proceedings, 931-935.

Sherrit, S., Wiederick, H., Mukherjee, B., \& Sayer, M. (21 de 8 de 1997). An accurate equivalent circuit for the unloaded piezoelectric vibrator in the thickness mode. Journal of Physics $d$ : applied physics.
Sirohi, J., \& Chopra, I. (April 2000). Fundamental Understanding of Piezoelectric Strain Sensors. Journal of Intelligent Material Systems And Structures, 11.

Sokhanvar, S. E. (2007). Investigating The Effect Of The Orthotropic Property Of Piezoelectric. Transactions of the CSME/ SCGM, 31 (1).

UNIMINUTO, D. D. (2016). Determinación de la energía producida en la relación a las dimensiones cerámicas piezoeléctricas de titanio de Barios para aplicaciones en ecosistemas inteligentes. Recuperado de http://www. uniminuto.edu/web/investigacionesp/proyectos

US, M. S. (1984). Piezoelectric Ceramic for Sonar Transducers (Hydrophones \& Projectors). us: Military Standard us.

Wang, J. J., Meng, V. X., Ma, Q., Xu, M. X., \& Chen, L. Q. (2010). Lattice, elastic, polarization, and electrostrictive properties of $\mathrm{BaTiO} 3$ from first-principles. Pennsylvania-usa: The American Institute of Physics.

Wingqvist, G. E. (2009). On The Applicability Of High Frequency Acoustic Shear Mode Biosensing InView Of Thickness Limitations Set By The Film Resonance. Biosensors and Bioelectronics, 3387-3390.

Wu, D., Tsai, Y., \& Yen, Y. (2003). Robust design of quartz crystal microbalance using finite element and Taguchi method. Sensors and Actuators, 92, 337-344.

Zalazara, M., \& Guarnieria, F. (2010). Análisis Y Evaluación Del Comportamiento De Sensores Piezoeléctricos. Mecánica Computacional, xxIx, 6665-6684. Recuperado de http://www. amcaonline.org.ar. 